www.nature.com/pj

\title{
Synthesis of grafted polysilsesquioxane by ring-opening polymerization of lactide and trimethylene carbonate
}

\author{
Mikihiro Kashio $^{1}$, Toshio Sugizaki ${ }^{1}$, Yorihisa Tanaka ${ }^{2}$, Shin-ichi Yamamoto ${ }^{2}$, Osamu Moriya ${ }^{2}$ \\ and Tadatomi Nishikubo ${ }^{3}$
}

The polysilsesquioxane (PSQ) containing 3-aminopropyl and phenyl groups (APSQ) was prepared from the corresponding trimethoxysilanes by co-condensation under basic conditions. The amino groups on APSQ were used as the initiator species for the graft polymerization of 1,3-trimethylene carbonate (TMC) and L-(-)-lactide (LA). The ring-opening polymerization of TMC and LA in the presence of the catalytic 1-trimethylsilylimidazole proceeded effectively to afford the PSQ with poly(TMC) and/or poly(LA) as the graft chains. The grafted PSQ containing poly(LA) showed the heat durability as an advantage of the hybrid polymer. In differential scanning calorimetry analysis, the grafted random copolymer of TMC and LA showed no clear endotherm corresponding to melting point $\left(T_{\mathrm{m}}\right)$. For the poly(LA)-grafted PSQ, the peak indicating $T_{\mathrm{m}}$ was observed in the first heating run, but the corresponding peak was very small in the second run.

Polymer Journal (2011) 43, 525-530; doi:10.1038/pj.2011.22; published online 30 March 2011

Keywords: graft polymerization; lactide; polysilsesquioxane; ring-opening polymerization; trimethylene carbonate

\section{INTRODUCTION}

The grafting of a polymeric component is an effective method for modifying polysilsesquioxane (PSQ), which mainly consists of T-type siloxane bonds. In the modification, the properties of the incorporated components are provided to the PSQs in addition to thermal stability and weatherability from the inorganic polysiloxane backbone. ${ }^{1-7}$ Therefore, graft polymerization from or onto PSQ is an effective method to create useful hybrid materials. Consequently, various combinations of polymeric components and PSQs have been investigated. ${ }^{8-12}$ We have previously investigated the grafting of the biodegradable polymers $\varepsilon$-caprolactone (CL) and L-(-)-lactide (LA) from the PSQ main chain containing phenyl and aminopropyl groups (APSQ). ${ }^{13,14}$ In thermal analyses of the resultant PSQs, low crystallinity of the polymeric components seems to be induced by the presence of the polysiloxane structure. This property is favorable for the use of grafted PSQs as practical hybrids. Furthermore, the PSQs containing polymerized CL and LA are expected to lead to new environmentally friendly materials that have applications in the biomedical field and as low-temperature adhesives. ${ }^{15}$ By contrast, the polymerized products of 1,3-trimethylene carbonate (TMC) usually exhibit low-temperature glass transitions from -25 to $-15^{\circ} \mathrm{C}$. This property is favorable for amorphous elastomers with high-tensile strength and flexibility. ${ }^{16-18}$ In addition, poly(TMC) is also degradable in vivo and in vitro in lipase solutions. Therefore, the copolymer combining poly(TMC) with poly(LA) is expected to be useful in the fields of medicine and environmental science. In fact, several investigations concerning the ring-opening copolymerization of LA and TMC, which is regarded as a softening additive, have already been presented. ${ }^{19,20}$ Taking these findings together, the grafting of poly(TMC) and poly(LA) from APSQ is likely to form a useful hybrid material.

The possibility of developing a new hybrid led us to attempt the ring-opening polymerization of TMC using 1,5,7-triazabicyclo[4,4,0]dec-7-ene, which was found to be a metal-free catalyst in our previous grafting of cyclic esters from APSQ. ${ }^{14}$ However, in the ring-opening polymerization conducted at $80^{\circ} \mathrm{C}$ with furfurylamine as an initiator, the researchers observed the formation of byproducts through decarboxylation. The analogous use of a strong base such as 1,8diazabicyclo[5.4.0]-7-undecene has been reported to be effective for the polymerization of TMC. On the basis of the findings of a wellperformed study of catalytic activity, a strong base generally affects both polymerization and depolymerization. ${ }^{21}$ This finding suggests that the strong base caused decarboxylation in our preliminary experiment. Other candidates for the catalyst, imidazole (IM) compounds, which allow the ring-opening polymerization of LA, have been considered. ${ }^{23}$ Furthermore, the efficient formation of linear carbonate bonds has been achieved through the substitution reaction of intermediary acylimidazole and silyl alkoxide. ${ }^{22}$ These results led us to examine the ring-opening polymerization with the use of IM derivatives as catalysts for grafting from PSQ. The synthetic route is

${ }^{1}$ Research Laboratory, Lintec Co., Warabi, Japan; ${ }^{2}$ Department of Applied Chemistry, National Defense Academy, Yokosuka, Japan and ${ }^{3}$ Department of Material and Life Chemistry, Faculty of Engineering, Kanagawa University, Rokkakubashi, Yokohama, Japan

Correspondence: Professor O Moriya, Department of Applied Chemistry, National Defense Academy, 1-10-20 Hashirimizu, Yokosuka, Kanagawa 239-8686, Japan.

E-mail: moriyaos@nda.ac.jp

Received 5 November 2010; revised 9 February 2011; accepted 10 February 2011; published online 30 March 2011 


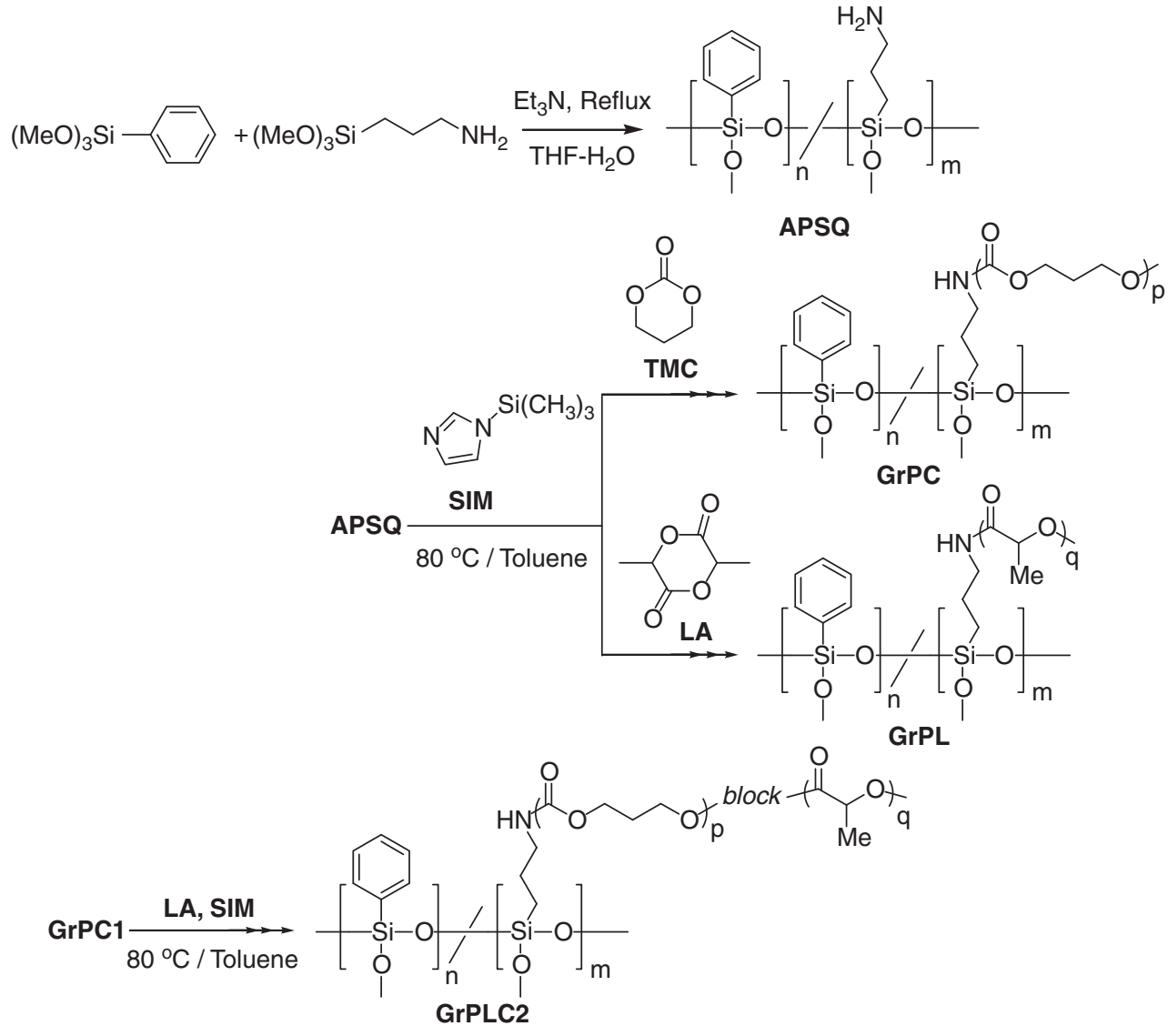

Scheme 1 Graft polymerization from APSQ (polysilsesquioxane containing 3-aminopropyl and phenyl groups). SIM, 1-trimethylsilylimidazole; THF, tetrahydrofuran; TMC, trimethylene carbonate.

shown in Scheme 1. In addition, because of interest in the thermal properties of the resultant hybrid materials, we conducted differential scanning calorimetry (DSC) and thermogravimetric analysis (TGA).

\section{EXPERIMENTAL PROCEDURE}

\section{General}

${ }^{1} \mathrm{H}$ - and ${ }^{13} \mathrm{C}$-nuclear magnetic resonance (NMR) spectra were obtained on JEOL AL-300 (JEOL, Akishima, Japan) and Bruker AVANCE-500 spectrometers (Bruker, Madison, WI, USA) in $\mathrm{CDCl}_{3}$ or $\mathrm{N}, \mathrm{N}$-dimethylformamide- $\mathrm{d}_{5}$. Infrared spectra were recorded on a JASCO FT-IR 230 (JASCO, Hachioji, Japan) using a $\mathrm{KBr}$ disk. Gel permeation chromatographic (GPC) analysis was carried out to estimate number-average molecular weight $\left(M_{\mathrm{n}}\right)$ and polydispersity $\left(M_{\mathrm{w}} / M_{\mathrm{n}}\right)$ on a Shimadzu LC-10VP chromatograph (Shimadzu, Kyoto, Japan) equipped with an evaporative light-scattering detector. The three columns, Shim-pack GPC-80MD, $-804 \mathrm{D}$ and $-802 \mathrm{D}$ (Shimadzu), were connected in series, and $\mathrm{N}, \mathrm{N}$-dimethylformamide was used as the eluent. Calibration was performed using poly(methyl methacrylate) standards. Toluene was refluxed over sodium metal and distilled. The monomer LA was recrystallized from ethyl acetate before use. Furfurylamine was distilled before use. Other reagents, including phenyltrimethoxysilane, (3aminopropyl)trimethoxysilane, IM, 1-trimethylsilylimidazole (SIM), TMC and diethyl ether, were used as supplied from commercial sources. APSQ was prepared by the reported method. ${ }^{14}$ The $M_{\mathrm{n}}$ and $M_{\mathrm{w}} / M_{\mathrm{n}}$ of the obtained APSQ were 1600 and 1.120, respectively. The contents of phenyl and aminopropyl groups, estimated by ${ }^{1} \mathrm{H}$-NMR data, were $1.27 \mathrm{mmol}_{\text {equiv }} \mathrm{g}^{-1}$ and $5.33 \mathrm{mmol} \mathrm{equiv}^{-1}$, respectively.

\section{Typical procedure for graft polymerization of TMC from APSQ} APSQ $(0.48 \mathrm{~g}, 0.61 \mathrm{mmol}$ equiv of amino group) and SIM $(0.14 \mathrm{~g}, 0.97 \mathrm{mmol})$ were charged in a flask equipped with a rubber septum under an argon atmosphere. TMC $(0.62 \mathrm{~g}, 6.07 \mathrm{mmol})$ was added to the flask using a syringe and the mixture was heated at $80^{\circ} \mathrm{C}$. After $20 \mathrm{~h}$ of polymerization, an excess amount of diethyl ether was added to the mixture. The viscous oil was collected by decanting and washed several times with diethyl ether. The resultant oil was dried at room temperature for $24 \mathrm{~h}$ under reduced pressure $(<2 \mathrm{~mm} \mathrm{Hg})$ to obtain the poly(TMC) grafted PSQ $(\mathrm{GrCl})(0.95 \mathrm{~g}, 86 \%$ yield based on weight and $67 \%$ yield based on phenyl group). The contents of the phenyl group and the monomer unit in $\mathrm{GrCl}$ were calculated from the peak areas observed in the ${ }^{1} \mathrm{H}-\mathrm{NMR}$ spectrum, in which nitromethane was used as an internal standard. The content of the amino group was estimated from that of the phenyl group by referring to the ratio of the groups in the starting APSQ; infrared (KBr) 3418 (weak), 2969 (weak), 1746 (strong, C=O), 1461 (weak), 1407 (weak), 1331 (weak), 1245 (strong, C-O), 1133 (medium), 1034 (medium, Si-O), 927 (weak), 791 (weak) $\mathrm{cm}^{-1}$; ${ }^{1} \mathrm{H} \mathrm{NMR}\left(\mathrm{CDCl}_{3}, 500 \mathrm{MHz}\right) \delta 2.04\left(\mathrm{~m},-\mathrm{CH}_{2}-\right)$, $4.23\left(\mathrm{~m}, \mathrm{O}-\mathrm{CH}_{2}-\right), 7.23-7.71\left(\mathrm{br},-\mathrm{C}_{6} \mathrm{H}_{5}\right) ;{ }^{13} \mathrm{C}$ NMR $\left(125 \mathrm{MHz}, \mathrm{CDCl}_{3}\right)$ $\delta 33.36\left(-\mathrm{CH}_{2}^{-}\right), 70.34\left(\mathrm{O}-\mathrm{CH}_{2}-\right), 133.69\left(-\mathrm{C}_{6} \mathrm{H}_{5}\right), 139.4\left(-\mathrm{C}_{6} \mathrm{H}_{5}\right), 160.55$ $(-\mathrm{C}=\mathrm{O}), \quad M_{\mathrm{n}}=4900 \mathrm{~g} \mathrm{~mol}^{-1}, \quad M_{\mathrm{w}} / M_{\mathrm{n}}=1.31, \quad$ phenyl group $=1.71 \mathrm{mmol}$ equiv $\mathrm{g}^{-1}$, aminopropyl group $=0.42 \mathrm{mmol}_{\text {equiv }}{ }^{-1}$ and $\mathrm{TMC}$ unit $=$ $5.67 \mathrm{mmol} \mathrm{equiv}^{-1}$.

Analogously, graft polymerizations using TMC and LA were carried out under the conditions shown in Tables 1 and 2. The content of the monomer unit was estimated from ${ }^{1} \mathrm{H}-\mathrm{NMR}$ data. In the estimation, the content of the lactic acid unit, shown in a previous report, ${ }^{14}$ was counted as the monomer unit from LA.

For the measurements of time versus conversion and conversion versus $M_{\mathrm{n}}$, the samples were collected using a syringe at the appropriate times during the polymerization and treated with a small amount of acetic acid. The samples were used for ${ }^{1} \mathrm{H}-\mathrm{NMR}$ measurements and GPC analysis without removal of the solvent. The results are depicted in Figures 1 and 2. 
Table 1 Ring-opening polymerization of TMC

\begin{tabular}{lclcrr}
\hline $\begin{array}{l}\text { Amine (feed molar } \\
\text { ratio of TMC/amino } \\
\text { group) }\end{array}$ & $\begin{array}{c}\text { Yield } \\
\%\end{array}$ & $\begin{array}{l}\text { Grafted } \\
P S Q\end{array}$ & $\begin{array}{c}\text { Ratio of the } \\
\text { monomer unit/ } \\
\text { amino group }\end{array}$ & $\begin{array}{c}\text { Calcd. } \\
\mathrm{M}_{n}{ }^{\mathrm{b}}\end{array}$ & $\mathrm{M}_{n}{ }^{\mathrm{c}}\left(\mathrm{M}_{w} \mathrm{M}_{n}\right)$ \\
\hline Furfurylamine (20) & 91 & FAC & 23 & 2600 & $3200(1.25)$ \\
APSQ (10) & 84 & GrPC1 & 14 & 4500 & $4900(1.31)$ \\
APSQ (20) & 73 & GrPC2 & 26 & 7000 & $6700(1.34)$ \\
APSQ (40) & 67 & GrPC3 & 37 & 9300 & $10700(1.35)$
\end{tabular}

Abbreviations: APSQ, polysilsesquioxane (PSQ) containing 3-aminopropyl and phenyl groups; $\mathrm{PSQ}$, polysilsesquioxane; TMC, trimethylene carbonate.

a Polymerization was carried out at $80^{\circ} \mathrm{C}$ under an argon atmosphere, in which the feed molar ratio of the 1-trimethylsilylimidazole/amino group was 1.5 and the concentration of TMC in toluene solution was $2.0[\mathrm{M}]$.

The contents of the unit and the group were calculated from ${ }^{1} \mathrm{H}$ nuclear magnetic resonance

spectral data, in which nitromethane was used as an internal standard.

'The values were based on gel permeation chromatography analysis, in which poly(methyl

methacrylate) standards were used.

dHomopolymer of TMC.

Table 2 Ring-opening polymerization of LA and TMCa

\begin{tabular}{llllll}
\hline $\begin{array}{l}\text { Monomer/amine (feed } \\
\text { molar ratio of }\end{array}$ & Grafted & $\begin{array}{c}\text { Yield } \\
\text { monomer/amino group) }\end{array}$ & $\begin{array}{c}\text { Ratio of the } \\
\text { monomer unit/ } \\
\text { amino group }\end{array}$ & $\begin{array}{c}\text { Calcd. } \\
\mathrm{M}_{n}{ }^{\mathrm{b}}\end{array}$ & $\mathrm{M}_{n}{ }^{\mathrm{c}}\left(\mathrm{M}_{w} \mathrm{M}_{n}\right)$ \\
\hline LA (20)/furfurylamine & FAL $^{\mathrm{d}}$ & 67 & 41 & 3000 & $3700(1.21)$ \\
LA (20)/APSQ & GrPL1 & 76 & 36 & 6900 & $8600(1.30)$ \\
LA (30)/APSQ & GrPL2 & 60 & 73 & 10800 & $13700(1.95)$ \\
LA (8), TMC (10)/APSQ & GrPLC1 & $23^{\mathrm{e}}$ & 8,2 & 3200 & $2700(1.25)$ \\
LA (30)/GrPC1 $^{f}$ & GrPLC2 & 84 & 44 & 10900 & $13300(1.34)$
\end{tabular}

Abbreviations: APSQ, polysilsesquioxane (PSQ) containing 3-aminopropyl and phenyl groups; LA, L-(-)-lactide; PSQ, polysilsesquioxane; TMC, trimethylene carbonate.

aPolymerization was carried out at $80^{\circ} \mathrm{C}$ under an argon atmosphere, in which the feed molar

ratio of 1-trimethylsilylimidazole/amino group was 1.5 and the concentration of TMC in toluene solution was $2.0[\mathrm{M}]$.

${ }^{\mathrm{D}}$ The contents of the unit and the group were calculated from ${ }^{1} \mathrm{H}$ nuclear magnetic resonance spectral data, in which nitromethane was used as an internal standard.

'The values were based on gel permeation chromatography analysis, in which poly(methyl methacrylate) standards were used.

dHomopolymer of LA.

${ }^{\mathrm{e} A}$ random copolymer of TMC and LA was grafted.

The estimated molar ratio of the TMC monomer unit/amino group in the starting GrPC was 14

\section{Thermal analysis}

TGA analysis was run using a Shimadzu DTG-60. The measurement was performed in air with $10 \mathrm{mg}$ of sample from 40 to $600^{\circ} \mathrm{C}$, for which the heating rate was $10^{\circ} \mathrm{C} \mathrm{min}^{-1}$.

DSC measurements were made on TA Instruments' Q2000 DSC (TA, New Castle, DE, USA). The purge gas was nitrogen at a flow rate of $50 \mathrm{ml} \mathrm{min}^{-1}$. The sample was kept for $5 \mathrm{~min}$ at $0^{\circ} \mathrm{C}$ before heating at a rate of $10^{\circ} \mathrm{C} \mathrm{min}-1$.

The TMC FA initiated poly(TMC) (FAC) and LA FA initiated poly(LA) (FAL) polymers, which were prepared with furfurylamine, were also used for the analyses.

\section{RESULTS AND DISCUSSION}

Polymerization of TMC from amino groups

The ring-opening polymerizations of TMC with furfurylamine using several IM catalysts, such as IM, SIM, 2-ethyl-5-methylimidazole and benzimidazole, were examined. The polymerizations were carried out in toluene solutions at $80^{\circ} \mathrm{C}$ with a monomer concentration of 2.0 $[\mathrm{M}]$ and a 1/1.5/20 feed mole ratio of the amino group/catalyst/TMC. The results of the preliminary experiments indicate that the IM derivatives, except for 2-ethyl-5-methylimidazole, have analogous catalytic activities for the polymerization of TMC. The polymerization behaviors using IM and SIM are depicted in Figures 1 and 2. When

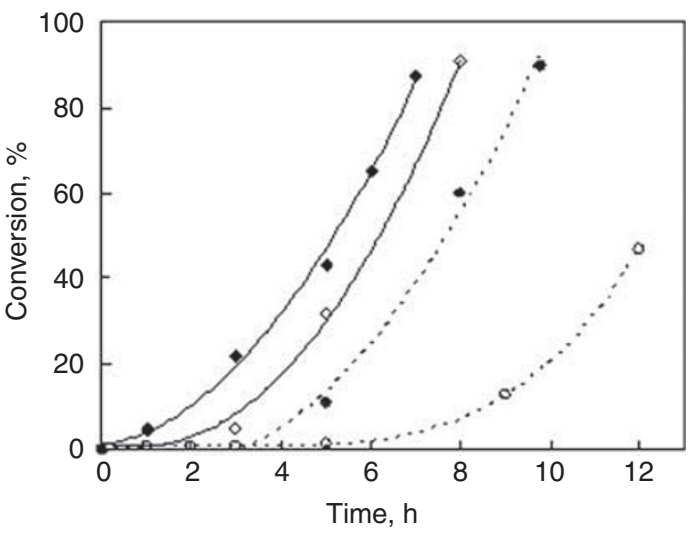

Figure 1 Time-conversion curves of polymerization of trimethylene carbonate at $80^{\circ} \mathrm{C}$ in toluene solution with imidazole $(\mathrm{IM})(\diamond)$, IM with FA $(\diamond)$, 1-trimethylsilylimidazole $(\mathrm{SIM})(O)$ and SIM with FA $(\bullet)$. FA, furfrylamine.

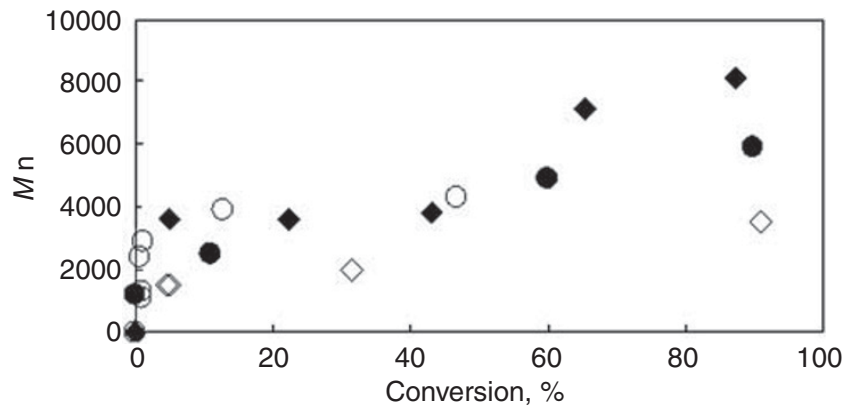

Figure 2 Conversion $M_{\mathrm{n}}$ curves of polymerization of trimethylene carbonate at $80^{\circ} \mathrm{C}$ in toluene solution with imidazole $(\mathrm{IM})(\diamond)$, IM with FA $(\diamond)$, 1-trimethylsilylimidazole (SIM) $(\bigcirc)$ and SIM with FA $(\bullet)$.

polymerization was carried out with IM without furfurylamine, the conversion of TMC began to increase after $2 \mathrm{~h}$, as shown in Figure 1. When using SIM, an induction period of ca $5 \mathrm{~h}$ was observed. The addition of furfurylamine shortened the period. The observations may be explained by a slow initiation in which a small amount of IM and the amine react to afford active species. This model suggests that the combination of IM and an amino group is required for the ringopening reaction. This interpretation is supported by findings in independent experiments, in which the substrate changes were followed using ${ }^{1} \mathrm{H}-\mathrm{NMR}$ analysis. In fact, the ring opening of TMC using IM or furfurylamine barely proceeded, even at $80^{\circ} \mathrm{C}$. As shown in Figure 2, the GPC measurements indicate unsteady increases of $M_{n}$ in the region of lower conversions. This result supports the speculation concerning the induction period mentioned above. In addition, it was found that the monomer, TMC, is consumed when ring-opening polymerization continues for $10 \mathrm{~h}$.

The ring-opening polymerization of TMC using IM, which was the most effective catalyst among the compounds examined in this work, was applied to the grafting from APSQ. However, a crosslinked solid that was insoluble in any versatile solvents was isolated. By contrast, the use of SIM allowed the preparation of the grafted PSQ, which was soluble in chloroform, toluene, acetone and $\mathrm{N}, \mathrm{N}$-dimethylformamide but insoluble in diethyl ether. When using IM, the pretreatment of APSQ with 1,1,1,3,3,3-hexamethyldisilazane avoided the formation of the crosslinked product. In other words, the use of SIM may protect the hydroxyl groups of the PSQ structure through trimethylsilylation. 
Although the exact structure has yet to be identified, the main chain of APSQ is thought to partially consist of ladder-like and incomplete cage-like polysiloxanes. Accordingly, APSQ should contain hydroxyl groups in the polysiloxane main chain. Furthermore, the trimethylsilylation of the end group after the ring opening of TMC may contribute to the selective chain growth in the polymerization.

On the basis of the experimental results mentioned above, SIM was used as the catalyst for the following synthesis of the grafted PSQs. The results of the graft polymerization of TMC from APSQ, in which the molar ratio of TMC/amino group was varied from 10 to 40, are listed in Table 1. The grafted PSQs (Table 1, GrPC1-3) were obtained in satisfactory yields. Even when using 40 equiv of TMC to the amino group, GrPC3 was obtained with a $67 \%$ yield. The monomer-units/ amino-group molar ratio of 37 in GrPC3, estimated by ${ }^{1} \mathrm{H}-\mathrm{NMR}$ spectral data, is almost the same as the feed molar ratios. Furthermore, the $M_{\mathrm{n}} \mathrm{s}$ of the products based on GPC analysis are understandable in light of those calculated from the contents of the monomer unit, although the detected values were somewhat larger than the calculated ones. For example, GrPC2 yielded a $M_{\mathrm{n}}$ of 6700 in GPC analysis, and the corresponding value is 7000 , which was calculated from the ratio of the TMC unit to one amino group in the graft chain. The polydispersities of the grafted PSQs range from 1.31 to 1.35 . The values are larger than the polydispersity of the starting APSQ, which has a value of 1.12. The homopolymer of TMC (FAC) was similarly isolated with a $91 \%$ yield and has a molar ratio of the monomer unit/ amino group of 23, reflecting the feed molar ratio of 20 .

\section{Polymerization of LA with TMC from APSQ}

For the ring-opening polymerization of LA, IM compounds are already known as effective catalysts. ${ }^{23}$ This fact indicates that SIM should also be a useful catalyst for the polymerization of LA. Consequently, the polymerization of LA from APSQ with SIM was examined under the conditions mentioned above. The results are listed in Table 2. At first, the polymerization of LA in the presence of SIM and furfurylamine was conducted to obtain the homopolymer (Table 2, FAL). FAL was isolated as an insoluble part of diethyl ether with a $67 \%$ yield. The ratio of the lactic-acid unit/furfurylamino group calculated from ${ }^{1} \mathrm{H}-\mathrm{NMR}$ spectral data is 41 , which is almost the same as the feed molar ratio of the substrate. The estimated molecular weight of FAL from the monomer-unit ratio is 3000 , and the $M_{\mathrm{n}}$ obtained by GPC measurement is 4700 . The grafting of poly(LA) using the SIM catalyst from APSQ also proceeded effectively. When the feed molar ratios of the LA/amino group were 20 and 30, the respective grafted PSQs (Table 2, GrPL1, GrPL2) contained ratios of 36 and 63 of the lactic acid unit. The estimated $M_{\mathrm{n}}$ values are 8600 for GrPL1 and 13700 for GrPL2 using GPC analysis, which are larger than the calculated values of 6900 and 10800 based on the monomerunit contents.

The copolymerization of LA and TMC was attempted for the grafting of a random copolymer. However, the product was obtained with a yield of only $23 \%$ (Table 2 , GrPLC1). The ${ }^{1} \mathrm{H}-\mathrm{NMR}$ spectral data indicate that the graft chain contains $8 \mathrm{U}$ of lactic acid and $2 \mathrm{U}$ of TMC to one amino group, although the numbers of the introduced lactic acid and TMC units were predicted to be 16 and 10 from the feed molar ratio of the monomers. The units due to LA were moderately grafted, but little incorporation of TMC unit into the graft chain was observed. An analogous copolymer composition was observed in the grafting of LA with $\varepsilon$-CL from APSQ. ${ }^{14}$ In this example, the content of the unit due to $\varepsilon$-CL in the graft chain was smaller than that due to LA. This difference demonstrates that LA acts as a retardant in the ring-opening copolymerization. At this stage, the

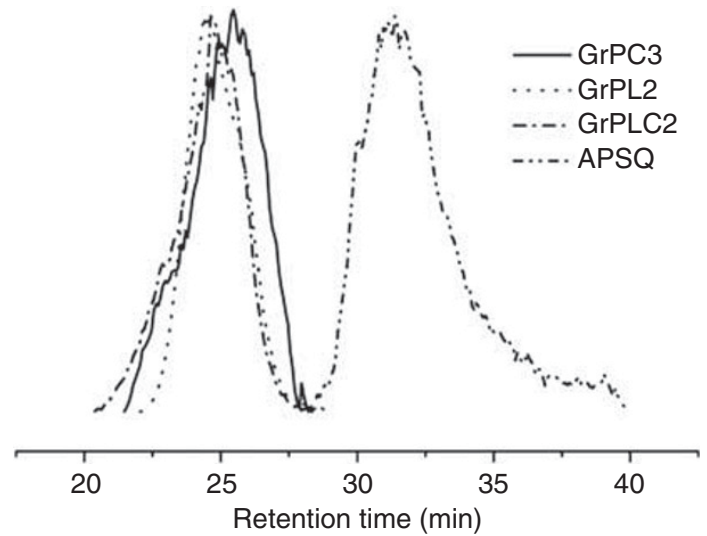

Figure $3 \mathrm{Gel}$ permeation chromatographic chromatograms of grafted polysilsesquioxanes. APSQ, polysilsesquioxane containing 3-aminopropyl and phenyl groups.

exact reason why the presence of LA prevents the normal polymerization of TMC has not been determined. However, the results suggest that the secondary alcohol of the end group of the polymerized LA is unfavorable for initiating the successive ring-opening polymerization of the other monomer. Thus, the introduction of a random copolymer of LA and TMC into APSQ using SIM is difficult.

The results obtained in the grafting of a random copolymer also suggest that the primary hydroxy end group of the TMC unit is preferable for the introduction of the other monomer units. Along with the above speculation, the introduction of the block copolymer of TMC and LA, which should change the thermal properties of the resulting PSQ, was tried. For the block copolymerization, the PSQ derivative, which contains 14 monomer units of TMC in the graft chain ( $\mathrm{GrPC1})$, was used as the macroinitiator. In the grafting of the second monomer, the content of the hydroxyl end groups due to the TMC units was regarded to be same as that of the amino group on GrPC1. The feed molar ratio of the LA/hydroxyl end group was 30. The introduction of the second monomer progressed efficiently to give PSQ with both monomer units of TMC and LA (Table 2, GrPLC2). The graft chain of GrPLC2 contained $44 \mathrm{U}$ of lactic acid to one amino group, which was calculated from the ${ }^{1} \mathrm{H}-\mathrm{NMR}$ spectral data. The estimated $M_{\mathrm{n}}$ by GPC measurement was 13300 , and the calculated molecular weight based on the content of the monomer units was 10900 . The difference between these values is reasonable in comparison with those shown when grafting poly(LA). The polydispersity of 1.34 recorded in GrPLC2 is similar to that of 1.31 in GrPC1.

The GPC chromatograms of several obtained compounds, such as APSQ, GrPC4, GrPL1 and GrPLC1, are shown in Figure 3. Each polymeric compound has a unimodal peak, suggesting that the graft polymerization onto APSQ proceeded using the IM catalyst. However, more detailed analyses are required to clarify that the ring-opening polymerizations of TMC and LA form the graft chains but not the corresponding homopolymers.

The formation of poly(TMC) and/or poly(LA) was confirmed by the spectral data, and the contents of the LA and TMC monomer units were estimated by ${ }^{1} \mathrm{H}-\mathrm{NMR}$ analysis measured with nitromethane as an internal standard. The spectrum of GrPLC1 is shown in Figure 4 as an example. In the spectrum, the signals due to the methylene protons are detected in the range from 1.3 to 1.6 p.p.m. After the grafting, the signal assigned to the methylene protons bonded to $\mathrm{Si}$ is hardly seen at 0.6 p.p.m., whereas a broad signal showing the presence of the benzene ring in APSQ is observed near 7.3 p.p.m. The peak area of the signals 


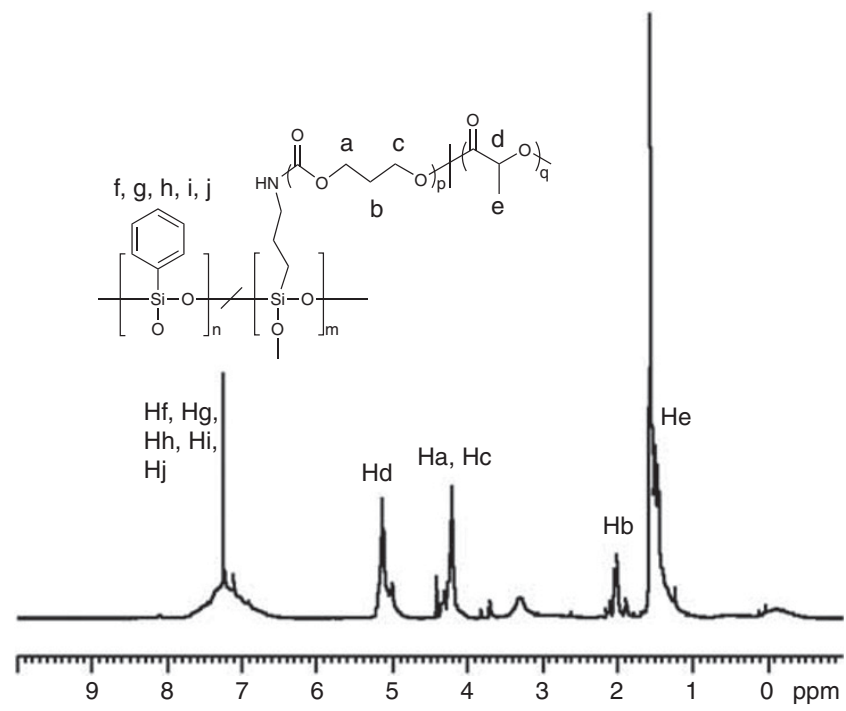

Figure $4{ }^{1} \mathrm{H}$ nuclear magnetic resonance spectrum of GrPCL1.

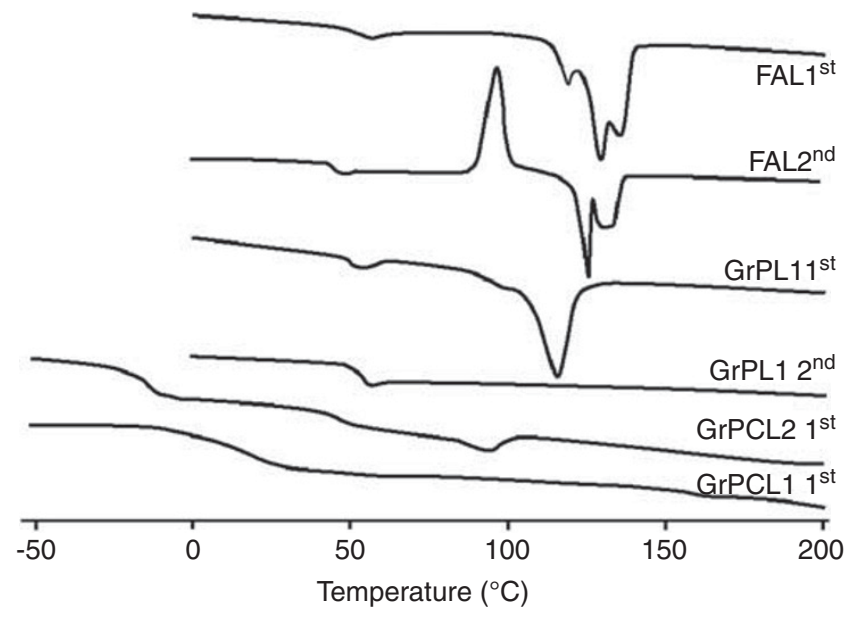

Figure 5 Differential scanning calorimetry curves of grafted polysilsesquioxanes.

assigned to the benzene ring was used to calculate the content of the amino group in the grafted APSQ. The characteristic signals due to methylene protons of the TMC unit were detected at 2.04 and 4.23 p.p.m. The latter signal was convenient for the calculation of the monomer-unit content. The presence of lactic acid units was identified by the signals at 1.56 and 5.14 p.p.m., which indicate the presence of methyl and methyne protons, respectively. The content of the lactic acid unit was readily calculated using the peak areas of those signals in comparison. The infrared and ${ }^{13} \mathrm{C}-\mathrm{NMR}$ spectral data of the grafted APSQs support the above analyses of the obtained polymers.

\section{Thermal properties}

To obtain information on the thermal properties of the grafted PSQs, DSC and TGA measurements were conducted. The results of DSC analysis are depicted in Figure 5 . The melting point $\left(T_{\mathrm{m}}\right)$ of poly(LA) is usually observed above $150{ }^{\circ} \mathrm{C} .{ }^{19}$ The homopolymer of LA, FAL, showed three peaks of the melting endotherm at ca $140{ }^{\circ} \mathrm{C}$ in the first heating run. The endotherm peaks were detected again in the second heating run. In the case of GrPL1, the peak at $120^{\circ} \mathrm{C}$ was observed in

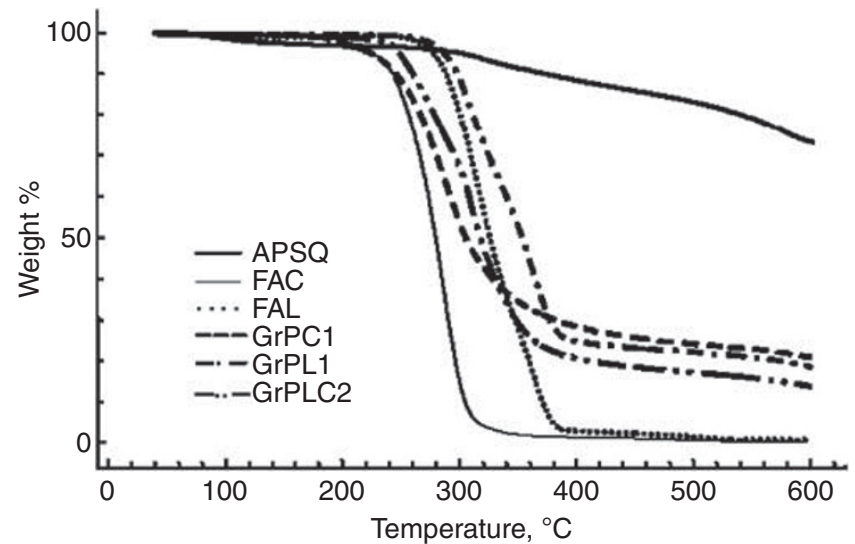

Figure 6 Thermogravimetric analysis curves of grafted polysilsesquioxanes. APSQ, polysilsesquioxane containing 3-aminopropyl and phenyl groups.

the first heating run, but the peak disappeared in the second heating. These findings suggest that the presence of the PSQ main chain induced a low crystallinity. The presence of poly(TMC) also affected the crystallization of poly(LA), as expected. ${ }^{14,24}$ The GrPLC1 curve shows an undetectable peak for $T_{\mathrm{m}}$, even in the first heating run. The results demonstrate that the incorporation of TMC units changes the crystallinity of the graft chains containing LA units. By contrast, almost all of the grafted PSQs show an endotherm peak around $55^{\circ} \mathrm{C}$, which is thought to be a glass-transition temperature $\left(T_{\mathrm{g}}\right)$. Poly(TMC) has been reported to have a lower $T_{\mathrm{g}}$, of less than $-20{ }^{\circ} \mathrm{C}^{24}$ Consequently, the observed $T_{\mathrm{g}} \mathrm{s}$ are thought to show those of the grafted LA polymer. The peak observed at $\mathrm{ca}-15^{\circ} \mathrm{C}$ seems to be the $T_{\mathrm{g}}$ of the grafted poly(TMC) in the case of GrPCL2.

The TGA curves are shown in Figure 6. A weight loss of APSQ began at $320{ }^{\circ} \mathrm{C}$ and is attributed to the decomposition of the aminopropyl and/or phenyl groups bonded to Si. The decompositions of FAC and FAL began at 240 and $280^{\circ} \mathrm{C}$, respectively. Nearly identical temperatures were recorded for the decompositions of $\mathrm{GrPCl}$ and GrPL1, respectively. In comparison with the starting temperatures of the decompositions, no obvious improvement of thermal stability due to the presence of the inorganic siloxane structure was seen. However, the temperature for a $10 \%$ weight loss of the samples to occur seems to indicate the sample's heat durability. A $10 \%$ weight loss of GrPL1 was observed at ca $325^{\circ} \mathrm{C}$, and a lower temperature of ca $310^{\circ} \mathrm{C}$ was recorded for FAL. Analogously, GrPC1 yielded a higher temperature of $260^{\circ} \mathrm{C}$ for $10 \%$ weight loss compared with $245^{\circ} \mathrm{C}$, which was recorded for FAC. The TMC polymer was degradable at a lower temperature than that of LA. Consequently, GrPLC2, containing both monomer units of TMC and LA in the graft chains, lost $10 \%$ weight at ca $268^{\circ} \mathrm{C}$, which is higher than that observed for GrPC1.

\section{CONCLUSION}

The PSQ containing phenyl and aminopropyl groups, APSQ, was used for the grafting of poly(LA) and/or poly(TMC) to afford new organicinorganic hybrid materials in which SIM was shown to be an effective catalyst for the ring-opening polymerization of the monomers. However, the IM catalyst was unsuitable for the preparation of a random copolymer of LA and TMC. To graft a copolymer from and/or onto APSQ, a block copolymer in which TMC had to be used as the first monomer was introduced.

The presence of TMC units caused the low crystallinity of the polymeric component of LA. Furthermore, the polysiloxane main 
chain seemed to reduce the crystallinity of the poly(LA) graft chain in addition to improving the heat durability. The investigation of the effects of the PSQ main chain on the thermal properties of the poly(LA) graft chain has progressed, and the results will be presented in the near future.

1 Baney, R. H. \& Cao, X. Silicon-Containing Polymers (eds Johns, R. G., Ando, W., Chojnowski, J.) (Kluwer: Dordrecht, 2000).

2 Baney, R. H., Itoh, M., Sakakibara, A. \& Suzuki, T. Silsesquioxanes. Chem. Rev. 95, 1409-1430 (1995).

3 Kondo, T., Yoshi, K., Horie, K. \& Itoh, M. Photoprobe study of siloxane polymers. 3. Local free volume of polymethylsilsesquioxane probed by photoisomerization of azobenzene. Macromolecules 33, 3650-3658 (2000).

4 Lee, J.-K., Char, K., Rhee, H.- W., Ro, H. W., Yoo, D. Y. \& Yoon, D. Y. Synthetic control of molecular weight and microstructure of processible poly(methylsilsesquioxane)s for low-dielectric thin film applications. Polymer 42, 9085-9089 (2001).

5 Liu, H., Xu, J., Li, Y., Li, B., Ma, J. \& Zhang, X. Fabrication and characterization of an organic-inorganic gradient surface made by polymethylsilsesquioxane (PMSQ). Macromol. Rapid. Commun. 27, 1603-1607 (2006).

6 Ciolacu, F. C. L., Choundhury, N. R., Dutta, N. \& Kosior, E. Molecular level stabilization of poly(ethylene terephthalate) with nanostructured open cage trisilanolisobutyl-POSS. Macromolecules 40, 265-272 (2007).

7 Kawakami, Y., Yamaguchi, K., Yokozawa, T., Serizawa, T., Hasegawa, M. \& Kabe, Y. Higher polyhedral silsesquioxane (POSS) cage by amine-catalyzed condensation of silanols and related siloxanes. Chem. Lett. 36, 792-793 (2007).

8 Pyun, J. \& Matyjaszewski, K. Synthesis of nanocomposite organic/inorganic hybrid materials using controlled living radical polymerization. Chem. Mater. 13, 3436-3448 (2001).

9 Kim, K.-M., Keum, D.- K. \& Chujo, Y. Organic-inorganic polymer hybrids using polyoxazoline initiated by functionalized silsesquioxane. Macromolecules 36, 867875 (2003).

10 Cardoen, G. \& Coughlin, E. B. Hemi-Telechelic polystyrene-poss copolymers as model systems for the study of well-defined inorganic/organic hybrid materials. Macromolecules 37, 5123-5126 (2004).
11 Fu, B. X., Lee, A. \& Haddad, T. S. Styrene-butadiene-styrene triblock copolymers modified with polyhedral oligomeric silsesquioxanes. Macromolecules 37, 5211-5218 (2004).

12 Ohno, K., Sugiyama, S., Koh, K., Tsujii, Y., Fukuda, T., Yamahiro, M., Oikawa, H., Yamamoto, Y., Ootake, N. \& Watanabe, K. Living radical polymerization by polyhedral oligomeric silsesquioxane-holding initiators: precision synthesis of tadpole-shaped organic/inorganic hybrid polymers. Macromolecules 37, 8517-8522 (2004).

13 Kashio, M., Sugizaki, T., Yamamoto, S., Matsuoka, T. \& Moriya, O. Ring-opening polymerization of $\varepsilon$-caprolactone by base catalyst for synthesis of grafted polysilsesquioxane. Polymer 49, 3250-3255 (2008).

14 Kashio, M., Sugizaki, T., Yamamoto, S. \& Moriya, O. Synthesis of grafted polysilsesquioxane by ring-opening polymerization of lactide. Polym. J. 41, 442-448 (2009).

$15 \mathrm{Xu}$, J. \& Shi, W. Synthesis and crystallization kinetics of silsesquioxane-based hybrid star poly(E-caprolactone). Polymer 47, 5161-5173 (2006).

16 Hori, Y., Gonda, Y., Takahashi, Y. \& Hagiwara, T. Ring-opening copolymerization of (R)$\beta$-butyrolactone with cyclic carbonates: new biodegradable poly(ester carbonate)s. Macromolecules 29, 804-806 (1996).

17 Ruckenstein, E. \& Yuan, Y. Molten ring-open copolymerization of L-lactide and cyclic trimethylene carbonate. J. Appl. Polym. Sci. 69, 1429-1434 (1998).

18 Zhang, Z., Zou, S. G., Vancso, G. J., Grijpma, G. J. \& Feijen, D. W. Enzymatic surface erosion of poly(trimethylene carbonate) films studied by atomic force microscopy. J. Biomacromolecules 6, 3404-3409 (2005).

19 Kim, J.- H., Lee, S. Y. \& Chung, D. J. Synthesis and properties of triblock copolymers from L-lactide and trimethylene carbonate. Polym. J. 32, 1056-1059 (2000).

20 Yang, J., Liu, F., Yang, L. \& Li, S. Hydrolytic and enzymatic degradation of poly(trimethylene carbonate-co-D, L-lactide) random copolymer with shape memory behavior. Eur. Polym. J. 46, 783-791 (2010).

21 Endo, T., Kakimoto, K., Ochiai, B. \& Nagai, D. Synthesis and chemical recycling of a polycarbonate obtained by anionic ring-opening polymerization of a bifunctional cyclic carbonate. Macromolecules 38, 8177-8182 (2005).

22 Bolton, D. H. \& Wooley, K. L. Synthesis of polycarbonates by a silicon-assisted alkoxy/carbonylimidazolide coupling reaction. J. Polym. Sci. Part A Polym. Chem. 35, 1133-1137 (1997).

23 Kricheldorf, H. R., Lomadze, N. \& Schwarz, G. Cyclic polylactides by imidazolecatalyzed polymerization of L-lactide. Macromolecules 41, 7812-7816 (2008).

24 Knight, P. T., Lee, K. M., Chung, T. \& Mather, P. T. PLGA-POSS end-linked networks with tailored degradation and shape memory behavior. Macromolecules 42 , 6596-6605 (2009). 